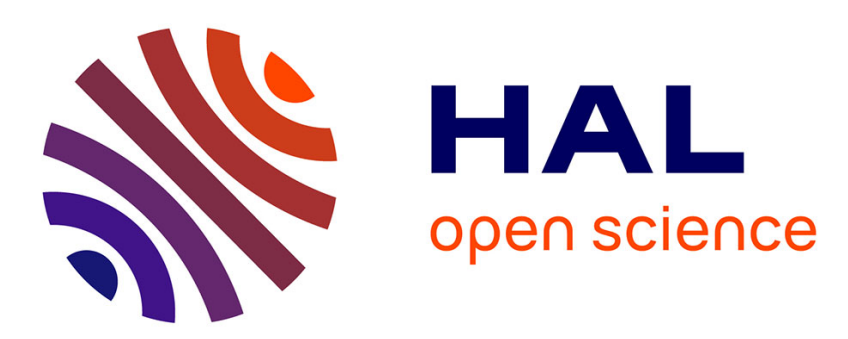

\title{
Mobilité des défauts à différentes températures dans les superalliages base Nickel
}

\author{
A. Coujou, N. Clément
}

\section{To cite this version:}

A. Coujou, N. Clément. Mobilité des défauts à différentes températures dans les superalliages base Nickel. Journal de Physique IV Proceedings, 1996, 06 (C2), pp.C2-89-C2-96. 10.1051/jp4:1996212 . jpa-00254190

\section{HAL Id: jpa-00254190 https://hal.science/jpa-00254190}

Submitted on 1 Jan 1996

HAL is a multi-disciplinary open access archive for the deposit and dissemination of scientific research documents, whether they are published or not. The documents may come from teaching and research institutions in France or abroad, or from public or private research centers.
L'archive ouverte pluridisciplinaire HAL, est destinée au dépôt et à la diffusion de documents scientifiques de niveau recherche, publiés ou non, émanant des établissements d'enseignement et de recherche français ou étrangers, des laboratoires publics ou privés. 


\title{
Mobilité des défauts à différentes températures dans les superalliages base Nickel
}

\author{
A. Coujou et N. Clément \\ CEMES-CNRS UPR 8011, 29 rue Jeanne Marvig, 31055 Toulouse, France
}

\begin{abstract}
Nickel Based superalloys are multiphased materials consisting in long range ordered $\gamma^{\prime}$ cuboïdal precipitates ( $\mathrm{Ll}_{2}$ strucrure) embeded in $\gamma$ matrix chanels. In situ deformation experiments in a Transmission Electron Microscop have been performed in order to study the defect mobility. This technique allows the observation of the dislocations dynamics under controlled conditions of stress and temperature. The microscopic mechanisms controling the deformation of each phase $\left(\gamma\right.$ and $\left.\gamma^{\prime}\right)$ have been analaysed. The role of these mechanisms in the global deformation of the material is discussed.
\end{abstract}

\section{INTRODUCTION}

Les superalliages base nickel ont une excellente tenue mécanique sur une large plage de températures. Ils ont de nombreuses applications industrielles : la température d'utilisation peut être basse dans les moteurs de fusées, moyenne dans certains outillages, et bien sur très élevée lorsque ce matériau est utilisé pour les pales de turbine des turboréacteurs. Ce sont des matériaux biphasés formés de précipités cuboidaux de phase durcissante $\gamma^{\prime}$, ordonnée à longue distance (structure L12), entourés de couloirs de matrice $\gamma$ de structure cfc (figure 1).

La technique de déformation "in situ" dans un MET utilisée ici est la seule qui permette l'observation de l'évolution de la microstructure et par conséquent de la mobilité des défauts sous contrainte et en température.

Nous allons distinguer les mécanismes élémentaires propres à chacune des phases $\gamma$ et $\gamma^{\prime}$, des mécanismes extrinsèques liés à la morphologie du matériau. Les résultats présentés sont ensuite discutés et comparés à ceux obtenus par d'autres techniques sur le même type de matériau, ainsi qu'aux modèles théoriques développés sur ce sujet.

\section{MOBILITE DES DEFAUTS DANS LA PHASE $\gamma^{\prime}$}

La phase $\gamma^{\prime}$ est ordonnée à longue distance (structure L12), et se caractérise par une anomalie de limite élastique. Pour étudier les mécanismes microscopiques responsables de cette propriété, des microéprouvettes monocristallines orientées [1] sont déformées à l'intérieur du microscope électronique de façon à étudier les glissements dans les plans octaédriques et cubiques. L'axe [001] privilégie les glissements octaédriques à l'exclusion du cubique, l'axe [110] déclenche les deux types de glissement de façon équivalente.

Il apparaît que dans cette phase, les mécanismes de dissociation de cœur des dislocations sont essentiels : les observations dynamiques en faisceau faible réalisées dans le domaine du pic de l'anomalie $\left(-196^{\circ} \mathrm{C}<\mathrm{T}\right.$ $<760^{\circ} \mathrm{C}$ ) montrent que la déformation est contrôlée par le déplacement des superdislocations vis. Leur mouvement dans les plans $\{111\}$ est saccadé. Le cœur des parties vis passe par des séries de double transition sessile-glissile. Entre 20 et $200^{\circ} \mathrm{C}$ l'amplitude de saut la plus probable est exactement égale à la largeur apparente de dissociation : on retrouve ainsi dans la phase $\gamma^{\prime}$, "les sauts de parois d'antiphase" analysés en détail sur l'intermétallique Ni3Al, alliage modèle de cette phase [2]. Un modèle en cours d'élaboration [3] permet d'expliquer l'essentiel des propriétés mécaniques de la phase L12 en glissement octaédrique entre 20 et $750^{\circ} \mathrm{C}$. 
A plus haute température $\left(\mathrm{T}>700^{\circ} \mathrm{C}\right)$, nous avons observé le cisaillement des précipités $\gamma^{\text {' }}$ par des superpartielles de Shockley de type $a / 3<112>$ traînant des défauts de surstructure. La figure 2 présente de tels mouvements : ces dislocations ont, sous contrainte, un mouvement visqueux $(\mathrm{v} \sim 40 \mathrm{~nm} / \mathrm{s})$, et se déplacent en restant parallèles à une direction $<110>$ [4].

$\mathrm{L}^{\prime}$ ensemble de ces résultats a permis de nuancer la notion de barrière de Kear-Wilsdorf admise jusque là. On sait maintenant que leur caractère sessile varie en fonction de la température et qu'elles peuvent, dans le domaine de l'anomalie, initier des glissements déviés sur les plans cubiques [5-9].

\section{MOBILITE DES DEFAUTS DANS LA PHASE $\gamma$}

Il existe peu de données sur les caractéristiques mécaniques et les mécanismes de déformation en fonction de la température de cette phase, probablement parce qu'aucun monocristal de $\gamma$ pur n'avait été jusqu'ici élaboré. Nous avons travaillé sur des monocristaux de phase $\gamma$ des superalliages MC2 et AM3 d'axe [001], spécialement élaborés à l'ONERA par P. Caron, sur la base des analyses de D. Blavette à la sonde atomique [10]. Par ailleurs entre $-196^{\circ} \mathrm{C}$ et $900^{\circ} \mathrm{C}$ des observations à l'échelle mésoscopique, en microscopie électronique à balayage, et microscopique, en microscopie électronique à transmission, montrent dans les échantillons déformés macroscopiquement, une localisation importante de la déformation qui se produit grâce au glissement d'empilements de dislocations. Au delà de $900^{\circ} \mathrm{C}$ par contre, la déformation devient homogène.

En tête de ces empilements les dislocations sont appariées (figure 3) Par déformation in situ la création et le mouvement de ces empilements ont pu être observés. A partir de la position des dislocations dans ces empilements nous avons pu déterminer la contrainte de friction qui s'oppose au déplacement des dislocations : elle est supérieure à la limite d'élasticité du matériau $\left(1,2 \sigma_{\mathrm{E}}<\sigma_{\mathrm{F}}<2,3 \sigma_{\mathrm{E}}\right)$ ce qui explique la présence de ces empilements qui, en multipliant la contrainte en tête, permettent le déplacement des dislocations.

Cette friction est due à la présence d'un "ordre local" que nous cherchons actuellement à qualifier. La présence de paires en tête d'empilements est la signature d'un tel ordre. Mais d'autres techniques (rayons $X$ et neutrons) sont nécessaires [12-15] pour évaluer les contributions de l'ordre à courte distance et d'un ordre à longue distance (type $\mathrm{L}_{2}$ ou $\mathrm{DO}_{22}$ ) dans chacun des alliages étudiés (CMSX2, MC2...). C'est la destruction de l'ordre local qui nécessite la contrainte élevée et qui est donc à l'origine de ces empilements. En conclusion, il apparaît que la phase $\gamma$ contribue à la résistance du biphasé de façon plus importante qu'on ne le considère habituellement, et que cette contribution varie suivant l'alliage considéré.

\section{MOBILITE DES DEFAUTS DANS LE BIPHASE.}

Les microéprouvettes monocristallines galbées de superalliage biphasé sont aussi déformées “ in situ " selon l'axe $\mathrm{X}=[100]$. Nous ne présenterons ici que les résultats obtenus à $20^{\circ} \mathrm{C}$ et à $850^{\circ} \mathrm{C}$.

$\mathrm{T}=20^{\circ} \mathrm{C}$

A cette température la déformation est très hétérogène. Nous avons pu observer les mécanismes élémentaires qui se produisent dans une petite bande de cisaillement composée d'une cinquantaine de dislocations. On constate que les deux principaux lieux de résistance à la propagation des dislocations sont les couloirs de matrice les plus étroits $(\mathrm{d} \sim 20 \mathrm{~nm})$, et les interfaces $\gamma / \gamma^{\prime}$ glissement des dislocations se produit grâce à la multiplication de contrainte que produisent de petits empilements (figures 4 et 5) de quelques dislocations (de 4 à 6 ). Le nombre de dislocations formant ces petits empilements s'adapte à la résistance de l'obstacle. Les précipités sont ensuite cisaillés par des superdislocations. Nous avons vu "in situ" les parties mixtes traverser ces précipités sans s'arrêter (figure 4) tandis que les parties vis avancent par bonds beaucoup plus lentement [11].

Plusieurs phénomènes physiques contribuent à la résistance exceptionnelle de ce matériau à cette température :

- l'ordre local de la solution solide qui freine la propagation des dislocations,

- la nécessité de donner une courbure importante aux dislocations à l'entrée des couloirs $\gamma$,

- le franchissement des interfaces,

- la dissociation des vis dans la phase $\gamma^{\prime}$. 
Les contraintes nécessaires au franchissement de chacun de ces lieux par la dislocation ont été évaluées [11].

$\mathrm{T}=850^{\circ} \mathrm{C}$

A cette température les dislocations simples de matrice se déplacent surtout (figure 6) dans les couloirs perpendiculaires à l'axe de traction ce qui montre que la contrainte interne due au désaccord paramétrique joue dans le $\mathrm{MC} 2$ un rôle dès $850^{\circ} \mathrm{C}$. Dans ces couloirs qui sont rapidement très écrouis, plusieurs systèmes de glissement participent à la déformation. Nous avons pour la première fois observé la multiplication des dislocations dans les couloirs $\gamma$. Elle a lieu (figure 7) grâce à la création de boucles (ouvertes ou fermées) par glissement dévié des parties vis des dislocations simples de matrice. Ce mode de multiplication couplé à la montée des parties mixtes sur les interfaces, permet un écrouissage tridimensionnel des couloirs qui se poursuit jusqu'à des taux de déformation élevés. Le glissement des dislocations dans les couloirs très écrouis, ainsi que la formation de boucles de très petite dimension, montrent qu'à cette température les dislocations concemées ont une grande flexibilité.

Nous avons aussi observé pour la première fois, dans les couloirs $\gamma$, le mouvement décorellé des dislocations partielles issues de la dissociation d'une dislocation simple de matrice [16].

Les super-Shockley qui cisaillent les précipités $\gamma^{\prime}$ ont un mouvement lent et visqueux et restent alignées selon des directions $\langle 110\rangle$. Un même précipité peut se trouver cisaillé plusieurs fois par des trains de super Schokley (figure 8) issues de dislocations simples de matrice. Après le passage d'une super-Shockley, il reste autour đu précipité une dislocation de Shockley. Ces faits sont en accord avec le modèle proposé par M. Condat et B. Décamps [17].

Nous n'avons pas observé une accélération des dislocations dans les ronds points ce qui semble montrer qu'il n'existe pas de concentration de contrainte sur les angles de précipités comme le prétendent certains calculs [18].

\section{DISCUSSION}

Pour le monophasé $\gamma$, en utilisant les dislocations (empilées ou isolées) comme sonde, nous avons pu estimer la contribution de cette phase à la résistance du biphasé.

Certains des résultats présentés ci-dessus à partir de la technique de déformation "in situ" confirment les observations déjà effectuées par d'autres équipes sur le matériau biphasé. Par exemple

- l'hétérogénéité de la déformation à $20^{\circ} \mathrm{C}$ a été observée "post-mortem" par Lisiecki [19] dans l'AM3 et M. Pessah et P. Caron dans le MC2 [20].

- la compétition entre contournement et cisaillement à basse température avait aussi été signalée par Sun par exemple [21].

- les mouvements décorellés des partielles dans certains couloirs de matrice $\gamma$ ont aussi été signalés par F. Saint Antonin et J. L. Strudel dans l'astroloy [22].

- le cisaillement des précipités par des superShockley trainant des SISF à haute température ont aussi été observés par de nombreux auteurs (Caron et al [23], Courbon et al [24], Condat et Descamps $[17], \ldots$...

Les observations dynamiques que nous présentons montrent que ces phénomènes existent bien sous contrainte et en température et permettent d'avoir une idée de leur cinétique. Elles ont aussi permis de proposer plusieurs mécanismes qui à notre connaissance n'avaient pas encore été signalés :

- celui de la multiplication des dislocations par boucles dans les couloirs de matrice à haute température,

- l'adaptation de la contrainte qui se produit grace aux petits empilements de dislocations qui se forment sur les différents lieux de résistance (entrée des couloirs, interfaces $\gamma / \gamma^{\prime}$ ).Ceci nous a conduit à réécrire les relations donnant les contraintes critiques de franchissemet de ces différents lieux pour tenir compte de la friction dans la phase $\gamma$ et de la présence de ces empilements $[11,25,26]$. 


\section{CONCLUSION}

L' observation « in situ » dans un MET de la dynamique des dislocations dans les phases $\gamma$ et $\gamma^{\prime} d^{\prime}$ 'un superalliage base Nickel a permis de mieux comprendre la contribution de chacune des phases à la résistance du biphasé.

L'observation de la mobilité des dislocations dans le matériau biphasé montre que plusieurs obstacles participent à la résistance exceptionnelle de ce matériau. Plusieurs mécanismes déjà proposés dans la littérature à partir d'observations « post mortem » ont été confirmés par ces observations. sous contrainte et en température. Quelques mécanismes nouveaux ont aussi été identifiés qui nous ont conduit à modifier les relations de la littérature pour introduire la résistance de la phase $\gamma$ ainsi que la multiplication de contrainte que créent la présence des petits empilements contre les interfaces $\gamma / \gamma^{\prime}$

\section{Références}

[ 1] Coujou A., Lours P., Roy N., Caillard D., Clément N., Acta Met. 38, nº 5 (1990) 825-837.

[ 2] Molénat G., Caillard D., Phil. Mag. A64, no 6 (1991) 1291-1317.

[ 3] Caillard D., Mat. Soc. Symp. Proc. 364 (1995) 17- 28.

[4] Lours P., Coujou A., De Mauduit B., Phil. Mag. A62, n² 2 (1990) 253-266.

[ 5] Clément N., Couret A., Caillard D., Phil. Mag. A64, n 3 (1991) 670-695.

[6] Clément N., Molénat G., Caillard D., Phil. Mag. A64, no 3 (1991) 697-708.

[ 7] Clément N., Caillard D., Lours P., Coujou A., Scripta Met. 23, n 4 (1989) 563.

[8] Clément N., Caillard D., Bénéteau A., Coujou A., Revue Phys. Appl. 23 (1988) 675.

[9] Lours P., Coujou A., Coulomb P., Acta Met. 39, n' 8 (1991) 1787-1797.

[10] Duval S., Chamberland S., Caron P. and Blavette D., Acta Metall. Mater. 42 (1994) 155.

[11] Benyoucef M., Clément N., Coujou A., sous presse Phil. Mag.

[12] Benyoucef M., Décamps B., Coujou A., Clément N., Phil. Mag. 71, 4 (1995) 707.

[13] Jouiad M., Clément N., Coujou A., à paraître.

[14] Glass R., Jouiad M., Caron P., Clément N. and Kirchner H.O.K., proposé à Acta Metall. Mater.

[15] Clément N., Coujou A., Calvayrac Y., Guillet F., Blavette D., Duval S., proposé à Microsc. Microanal. Microstruct.

[16] Coujou A., Benyoucef M., Saint Antonin F., Strudel J.L., Clément N., en préparation.

[17] Condat M. and Décamps B., Scripta Met. 21 (1987) 607.

[18] Pollock T.M., , and Argon A.S., Acta Met. 1 (1992).

[19] Lisiecki B., 1992, Thèse de l'Université Paris VI.

[20] Pessah M., 1994, Thèse de l'Université Paris VI.

[21] Sun Y., 1990, Thesis University Oxford.

[22] Saint Antonin F., 1991, Thèse Ecole des Mines de Paris.

[23] Caron P., Khan T, et Veyssière P., 1988, Phil. Mag. A6 (1988) 859.

[24] Courbon J., 1990, Thèse Institut National Polytechnique de Grenoble.

[25] Coujou A., Benyoucef M., Clément N., Solid State communication (1993) 345-352.

[26] Benyoucef M., Clément N., Coujou A., Mat. Science and Engineering A164 (1993) 401-406. 


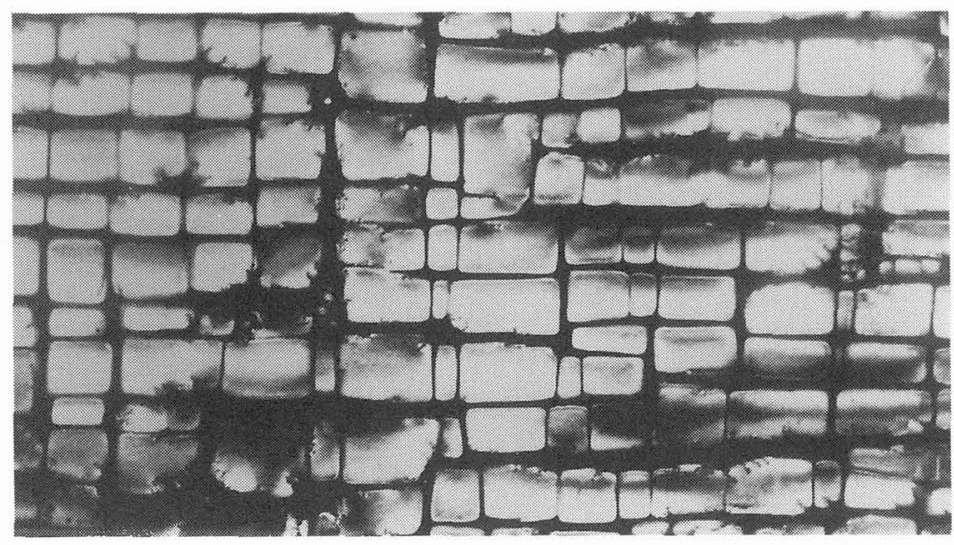

Figure 1 : Morphologie du superalliage $\mathrm{MC} 2$
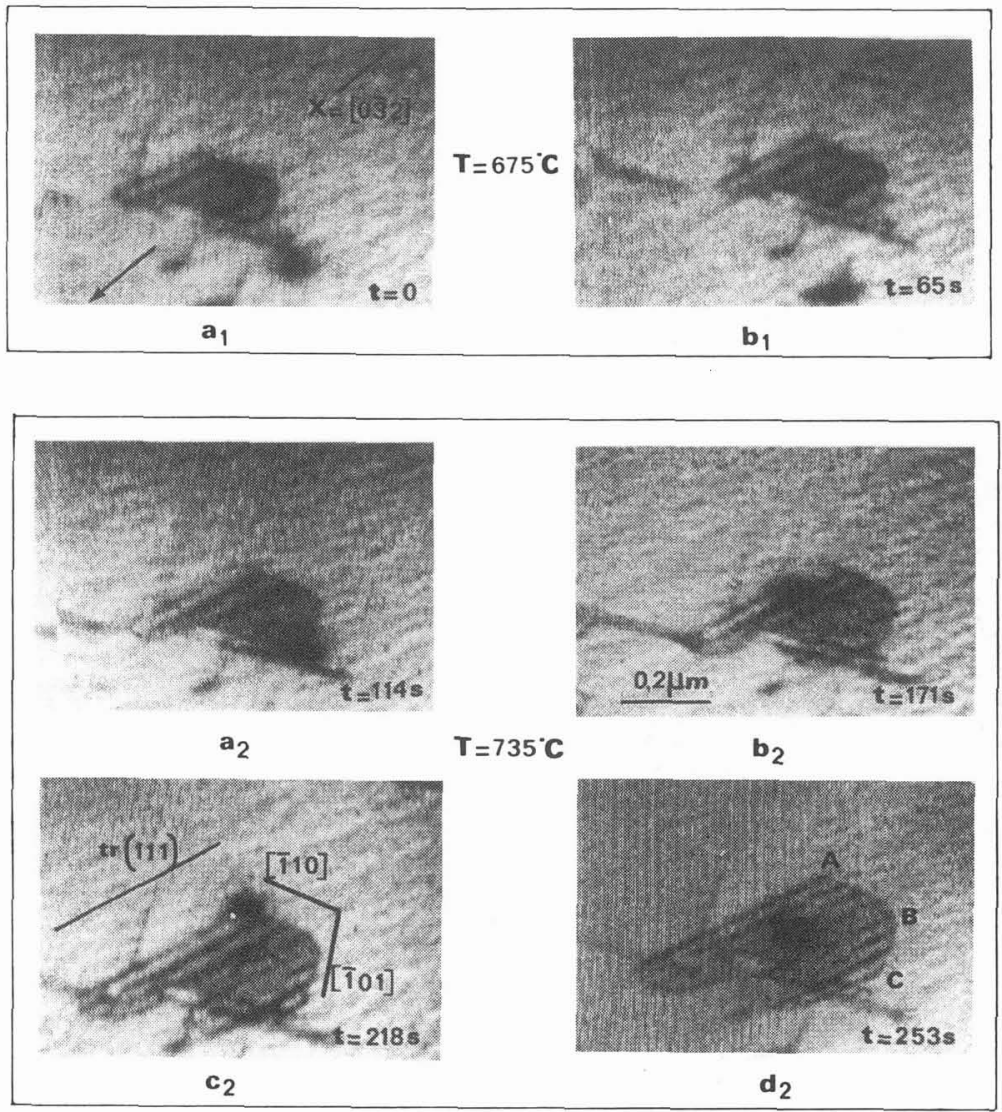

Figure 2 : Observation « in situ » du glissement de super-Shockleys à $675^{\circ} \mathrm{C}$ ) (clichés $\mathrm{a}_{1}$ et $\mathrm{b}_{1}$ ) et à $735^{\circ} \mathrm{C}\left(\mathrm{clichés} \mathrm{a}_{2}, \mathrm{~b}_{2}, \mathrm{c}_{2}\right.$ et $d_{2}$ ). 


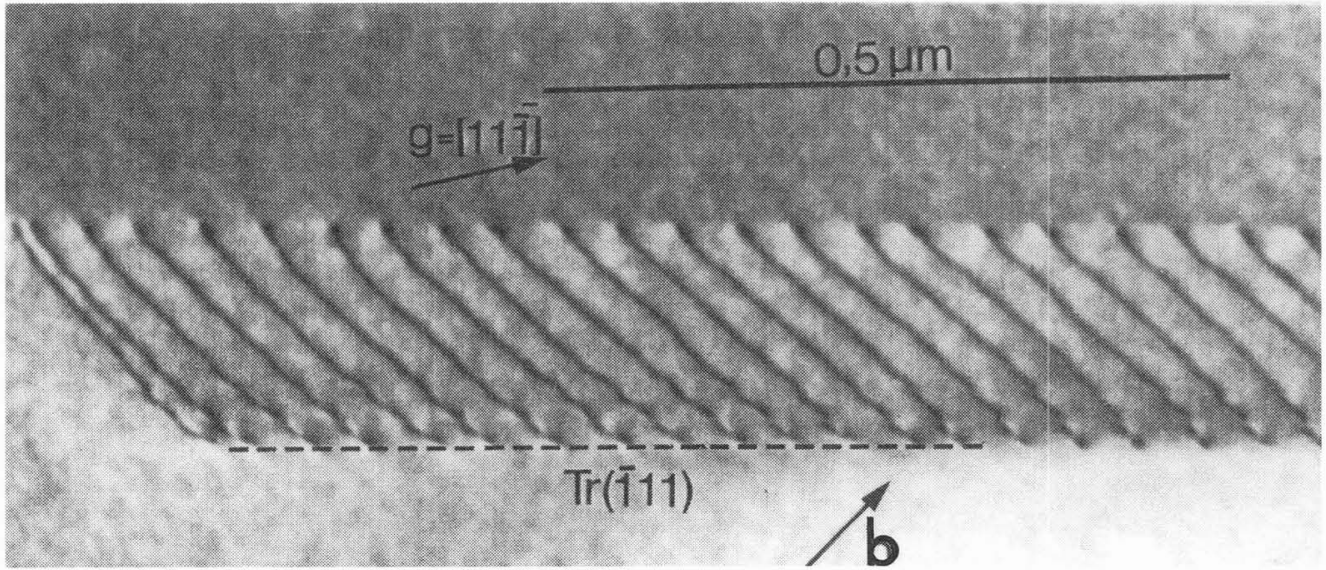

Figure 3 : Empilement de dislocations dans la phase $\gamma$ du superalliage MC2. Les deux dislocations de tête sont appariées à $20^{\circ} \mathrm{C}$.
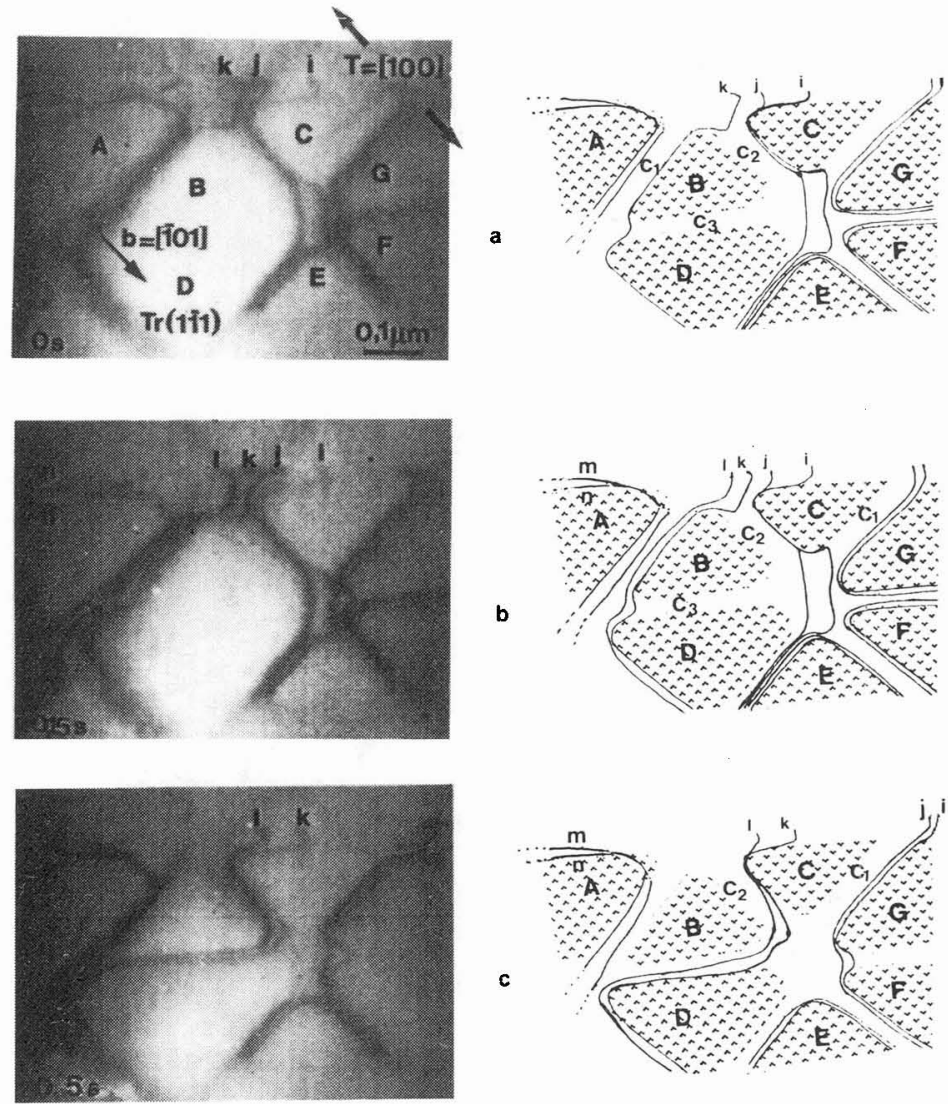

Figure 4 : Séquence de déformation « in situ » du superalliage biohasé MC2 à $20^{\circ} \mathrm{C}$. (a) état initial, (b) état intermédiaire : formation d'un empilcment de dislocations contre le précipité noté B, (c) état final 


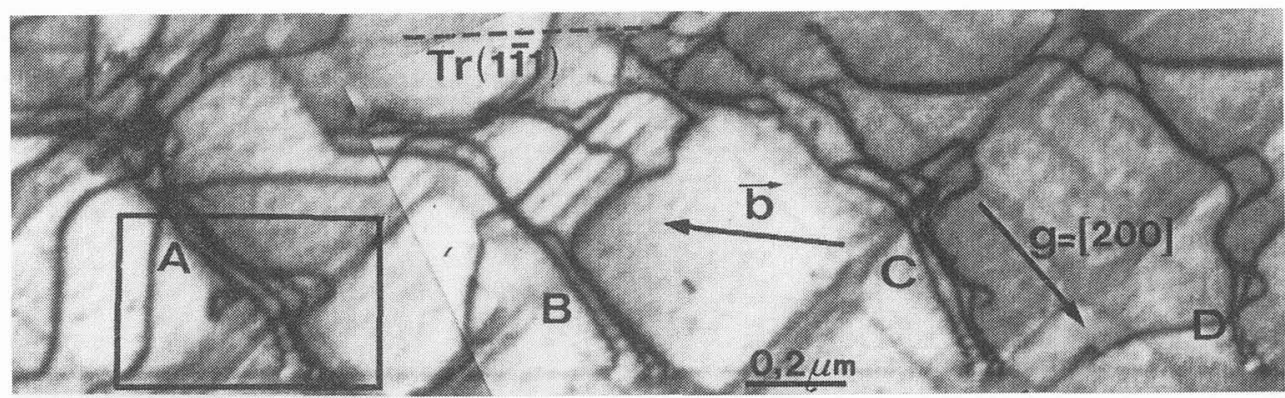

Figure 5 : Observation « post mortem » de la présence d'empilements de dislocations contre les interfaces $\gamma / \gamma^{\prime}$ à $20^{\circ} \mathrm{C}$.

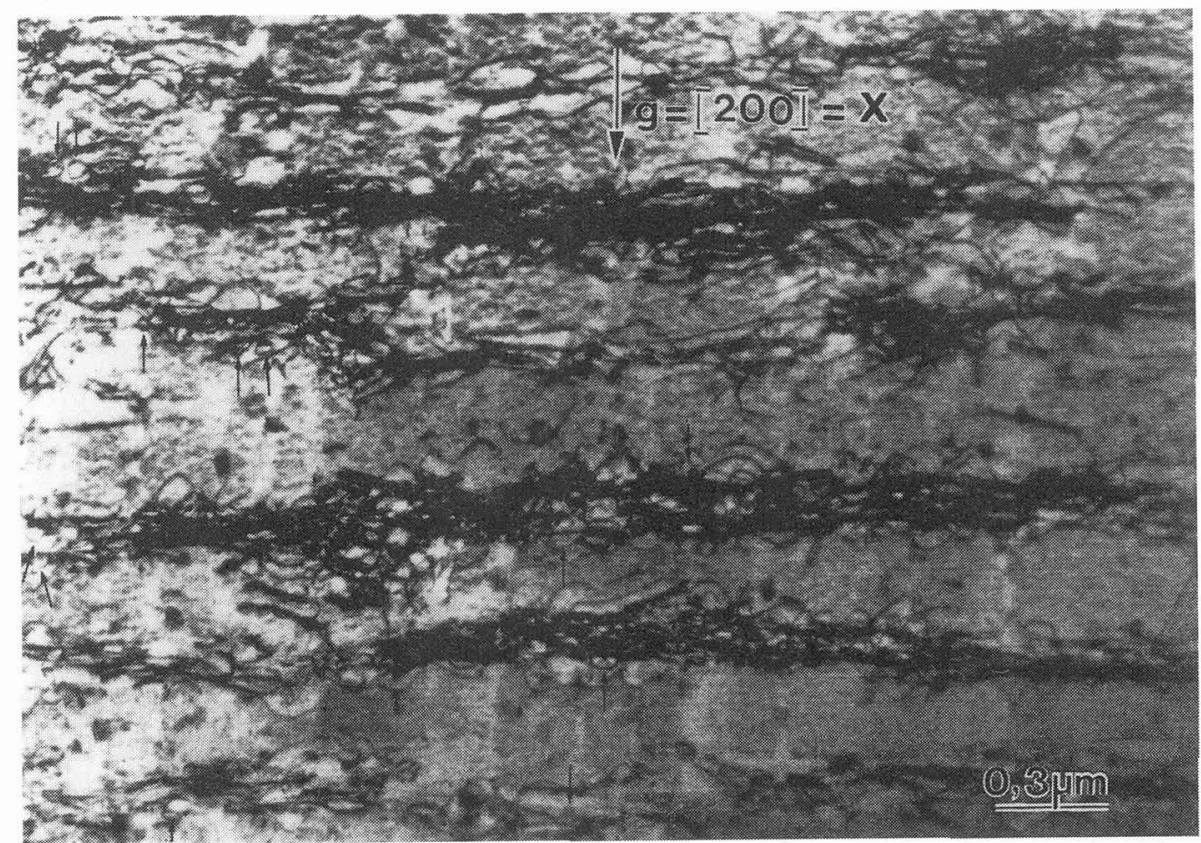

Figure 6: Ecrouissage des couloirs perpendiculaires à l'axe de traction à $850^{\circ} \mathrm{C}$ (observations post mortem). 


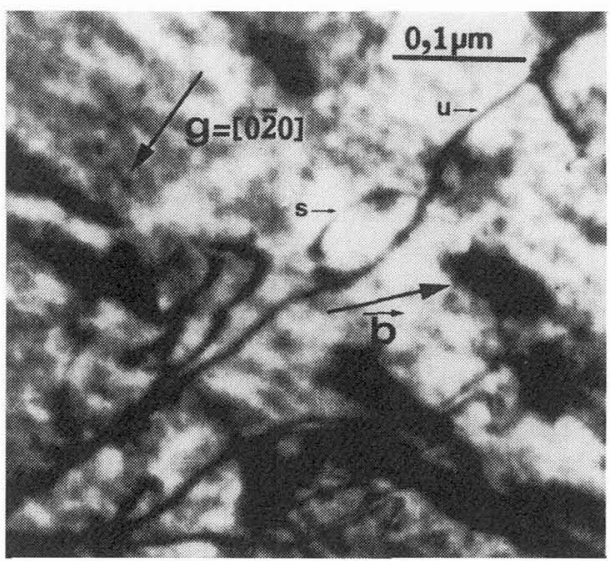

a

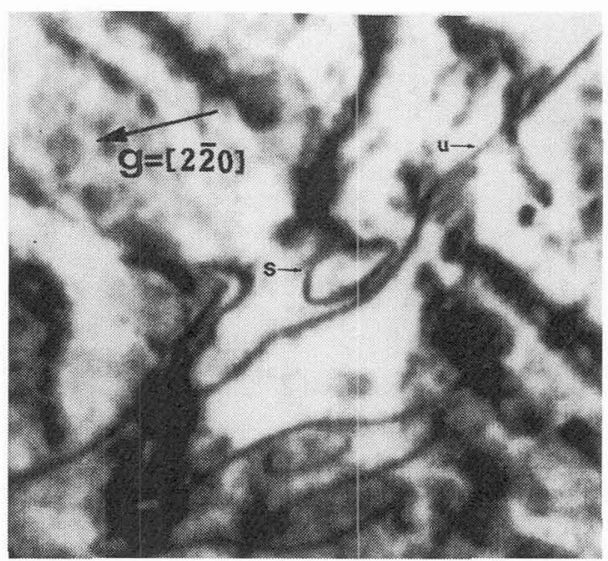

b

Figure 7 : Observation « post mortem » de la présence de boucles dans les couloirs de phase $\gamma$ à $850^{\circ} \mathrm{C}$.

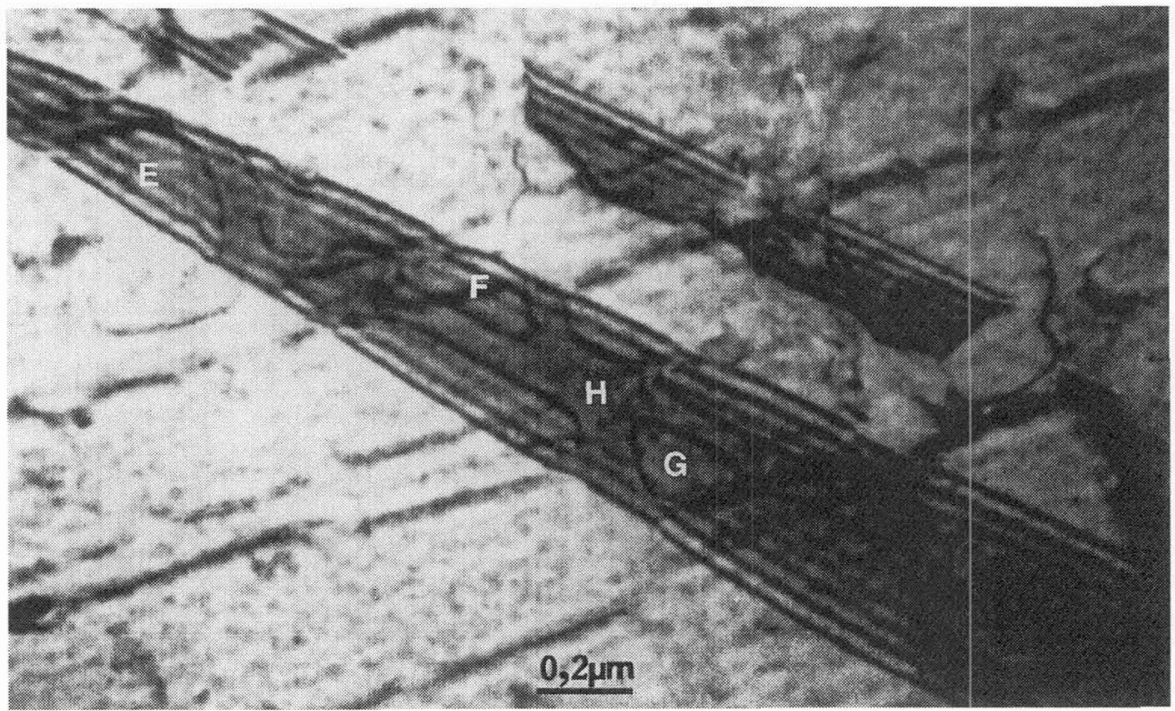

Figure 8: Observation «post mortem » du cisaillement des précipités $\gamma^{\prime}$ par des « super-Shockleys ». 